\title{
Making your next move: Dorsolateral prefrontal cortex and planning a sequence of actions in freely moving monkeys
}

\author{
JAE-WOOK RYOU and FRASER A. W. WILSON \\ University of Arizona, Tucson, Arizona
}

\begin{abstract}
Prefrontal damage disrupts planning, as measured by disorders of the activities of daily living (Humphreys \& Forde, 1998; Shallice \& Burgess, 1991). In a monkey model of this form of planning, a variant of the delayed alternation task was performed by freely moving monkeys. In a $16 \times 16$-ft. testing room, four feeders were located in the middle of each wall. In the north task, monkeys alternated between feeders: west-north-east-north-west, and so forth. In the south task, the alternation sequence was east-south-west-south-east, and so forth. Neuronal activity was recorded during walking along the eight paths, constituting the north and south tasks. To succeed, monkeys had to memorize the alternation rule and monitor both their place in the sequence and the previously made spatially directed action before deciding to walk to a new location to the left or right of the current location. Responsive dorsolateral prefrontal neurons are strikingly selective. Sustained neuronal activity reflects the spatial direction of an ongoing or upcoming response. It is important that such selective responses occur in one but not both tasks, even though the movements are exactly the same in both tasks and at each location. We suggest that selective neuronal activity is tuned through learning and reflects the fundamental units of a planning mechanism: Individual neurons encode specific components of a sequence of behavioral actions and their temporal order. Populations of such neurons represent all the steps necessary to perform the north and south tasks. The sustained activity of these neurons suggests that planning and working memory mechanisms are integrated.
\end{abstract}

Behavioral tasks that test spatial memory-the delayed alternation (DA) and delayed response (DR) tasks - have been the source of research into the cognitive functions of nonhuman primates for about 80 years (Jacobsen, 1935). Frontal lobectomy and, especially, limited resections of the dorsolateral prefrontal cortex (dlPFC), produce profound deficits in the abilities of monkeys to perform DA and DR tasks (Butters \& Pandya, 1969; Goldman \& Rosvold, 1970; Gross \& Weiskrantz, 1962; Mishkin, 1957). Jacobsen's interpretation of these deficits was that the brain damage impaired spatial memory, but this interpretation has never achieved consensus. For example, Malmo (1942) raised the possibility that the deficit was an attentional disorder. Subsequently, many studies have addressed different aspects of the role of the dlPFC in cognition, and Patricia Goldman-Rakic's work has been at the forefront of these efforts. Without question, her research has been instrumental in providing perspectives that allow the integration of basic research in monkeys with concepts of the normal function of the prefrontal

This research was supported by Whitehall Foundation Grant A89-04 and NIH Grant MH58415. We thank Byoung-Hoon Kim and Seung-Lark Lim for assistance with data acquisition. We also appreciate the support and encouragement given by Patricia Goldman-Rakic. Correspondence concerning this article should be addressed to J.-W. Ryou, Department of Neurology and Neuroscience, Weill Medical College, Cornell University, 1300 York Avenue, New York, NY 10021 (e-mail: jwryou@hotmail.com). cortex as well as its dysfunction in schizophrenia (e.g., Sereno \& Holzman, 1995). The DA and DR tasks have been central to research in monkeys (Goldman \& Rosvold, 1970; Levy \& Goldman-Rakic, 2000), and GoldmanRakic has continued to champion the view that the dIPFC is an essential component of spatial memory mechanisms, a concept that has become largely synonymous with spatial working memory (Baddeley, 1996).

Despite the robust and deleterious effect of dlPFC damage on DA/DR tasks in monkeys, it is not straightforward to reconcile these deficits with the effect of prefrontal damage in humans. Neurological studies tend to emphasize the loss of cognitive functions, such as the ability to plan (Stuss \& Benson, 1986), and many studies show that memory (both spatial and nonspatial) functions tend to remain intact in people with prefrontal damage. There are many reasons for the discrepancies between monkeys and humans, and we find two reasons that are compelling. First, brain damage in humans tends to be unilateral and does not respect anatomical boundaries, whereas experimental lesions in monkeys are usually bilateral and restricted to specific cortical territories. A second suggestion is that the frontal lobes occupy a large proportion of all cortical territories in both monkeys and humans, and of course in humans these territories are particularly large and presumably more specialized than in old-world rhesus monkeys. Anatomical studies (Cavada \& Goldman-Rakic, 1989; Dombrowski, Hilgetag, \& Bar- 
bas, 2001; Rajkowska \& Goldman-Rakic, 1995) attest to the multiplicity of anatomically distinct regions within the frontal lobes. Thus it is possible that the larger brains of human primates have functions that compensate for the loss of tissue that supports performance of the DA/DR tasks. These arguments, however, do not completely dispel the findings in which patients with prefrontal damage are unimpaired in the DA/DR tasks (see D'Esposito \& Postle, 1999, for a recent review), and these discrepancies need to be resolved.

Is the dIPFC in rhesus monkeys functionally different from that of humans? While acknowledging that humans probably have additional functions in their very much larger frontal lobes, we assume that some core functions are common to both monkeys and humans. Discrepancies are likely to arise in part because of the fundamentally different tasks used to test monkeys and humans. For example, Malmo's (1942) work suggests that the distracting sound of the screen door in the Wisconsin testing apparatus is important for the appearance of the DA/DR deficit in monkeys, and this should be tested in both monkeys and humans. In fact, when differences between tasks in humans and monkeys are minimized and functionality is probed with neurophysiological and imaging techniques, it is possible to conclude that similar neural mechanisms are engaged in both humans and monkeys (Courtney, Ungerleider, Keil, \& Haxby, 1997; Funahashi, Bruce, \& Goldman-Rakic, 1989; Ó Scalaidhe, Wilson, \& Goldman-Rakic, 1999; Smith \& Jonides, 1999). Of course, correlations between brain activity, cognition, and behavior do not necessarily result in the identification of the function that is critically dependent upon the prefrontal cortex. This is true for research in both human and nonhuman primates. However, we believe that progress in identifying dlPFC functions will be made by developing behavioral tasks for monkeys that are known to produce deficits in people with prefrontal damage. Accordingly, our recent efforts are aimed at developing methods for extending the DA/DR tasks into paradigms that are better measures of planning.

To this end, we were influenced by decades of observations of the effects of prefrontal brain damage on behavior. Penfield and Evans (1935, cited in Stuss \& Benson, 1986) observed that humans' ability to carry out a series of planned actions was disrupted by prefrontal damage. Inability to generate and execute a plan is impaired even when patients are able to perform well on tasks that are pure measures of discrete cognitive functions. The notion of "planning" can include very simple tasks (Humphreys \& Forde, 1998; Schwartz, Reed, Montgomery, Palmer, \& Mayer, 1991) that involve a series of actions that result in a goal, and perhaps the hallmark of prefrontal dysfunction is the disruption of everyday activities with apparent preservation of intellect. In their account of planning disorders, Shallice and Burgess (1991) argued that their subjects had preserved motivational and specialpurpose cognitive or memory processes and that their fundamental deficit was in the executive processes that allowed a course of planned actions to be implemented.
In this article, we describe a behavioral paradigm that we believe is able to probe working memory and planning in monkeys in a relatively natural setting. To this end, we developed the means to test freely moving monkeys (FMM; Ma, Ryou, Kim, \& Wilson, 2003) in a version of the DA task in which the monkeys were able to forage by walking to different locations in a testing laboratory. We accompanied this FMM DA task by recordings from single neurons during the behavioral testing. Our studies were influenced by neuropsychological work in people who report that frontal lobe damage produces impairments in planning and execution of activities such as shopping trips (Shallice \& Burgess, 1991), which are typical of daily living. We also wanted to adhere to principles advocated by Della Malva, Stuss, D'Alton, and Willmer (1993), who hypothesized that

to tap frontal resources, our task should include two essential components: on-line monitoring (where alternative courses of action exist and must be evaluated against ongoing feedback and ultimate goals); and intervention of a specific executive process within a sequencing context. (pp. 363)

\section{METHOD}

\section{Laboratory}

The testing room $(4.9 \mathrm{~m}$ wide $\times 5.5 \mathrm{~m}$ long $\times 3.5 \mathrm{~m}$ high $)$ was purpose-built for FMM experiments (Figure 1A). Along each wall is a feeder $(0.5 \mathrm{~m} \mathrm{high})$, at the front of which is a $5 \times 5 \mathrm{~cm}$ lid that needs to be raised, triggering a computer to deliver a piece of food (Wilson, Kim, Ryou, \& Ma, in press). The opening and closing of the feeder lids were detected by computer, and timestamps of these events were entered into a data file so that neuronal firing on each trial type could be aligned to critical task events for graphical display and statistical analysis. In the center of the room was a post (18 in. high) with a rotating spindle at the top. Mounted on the spindle was a housing that contained a retractable dog leash (Flexi Classic 1,5-m cord), which was connected to a collar worn by the monkey; as the monkey walked around the room, the spindle rotated. This leash was sufficiently long to allow the monkeys to comfortably sit and forage at the four feeders but not long enough to allow them to climb the feeders.

Curtains running behind the feeders reduced the asymmetry of the room so that it was effectively a square $(4.9 \times 4.9 \mathrm{~m})$. The curtains also made the testing room largely featureless, although the northeast door could be seen under the curtains, and a grill in the floor was located in the southeast quadrant of the room. Fluorescent lights were concealed in soffits close to the ceiling along all four walls. The entire room was painted gray-green. A chamber $(1 \mathrm{~m}$ wide $\times 1 \mathrm{~m}$ long $\times 0.7 \mathrm{~m}$ deep) was located in the center of the room's ceiling. It contained a color video camera (American Dynamics ADC762 1/3 in.) directed vertically down to image the monkeys' behavior, and an Air Flyte 72 channel slip-ring commutator (modified by Dragonfly Research and Development, Inc.) carried neurophysiological signals to power amplifiers in an adjacent control room. Investigators monitored equipment and the monkeys' behavior from the control room.

\section{Behavioral tasks}

Memory-guided DA sequence task. In this task, the monkeys alternated between three of the four feeders in a stereotyped sequence (Figure 1B). There were two versions of this task. In the north task, the monkeys alternated in the following way: west-north-eastnorth-west, and so forth. In the south task, the monkeys alternated 


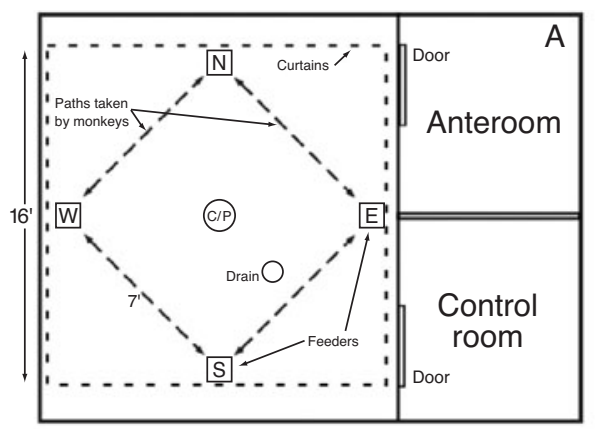

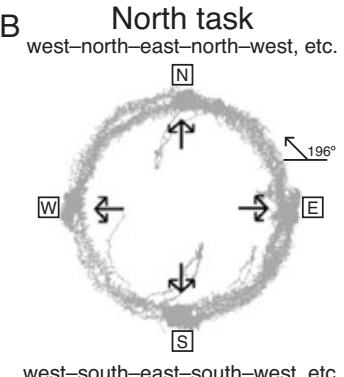

west-south-east-south-west, etc. South task

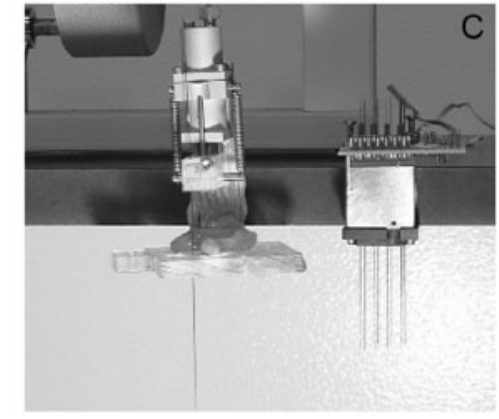

North

East

West

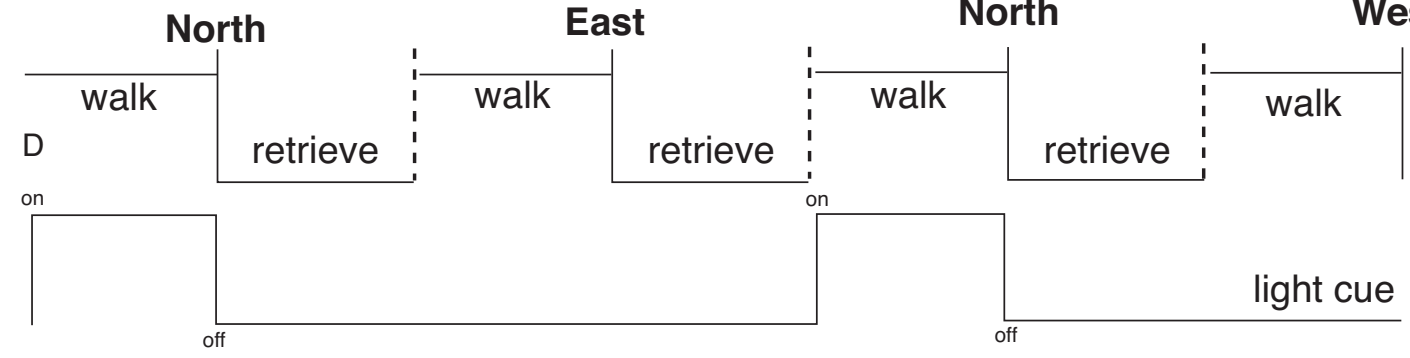

Figure 1. The freely moving monkey (FMM) testing room, plots of movement paths, and microdrive. (A) Schematic drawing of the testing and control room. (B) Schematic of the sequential organization of the delayed alternation (DA) task. At each choice point, a decision had to be made to turn left or right (135 $5^{\circ}$ minimum). Traces (grayscale) show plots of the monkey's position in the room during recordings of the neuron shown in Figure 4A. Pathways were usually restricted to the shortest distances (approximately $2.1 \mathrm{~m}$ ) between the feeders; there were very few excursions into the middle of the room. Movements between the feeders were very stereotyped. (C) Comparison of two microdrives. On the left is a conventional hydraulic microdrive. The device is top-heavy and unstable during head movements and walking. On the right is a custom-designed microdrive $(22 \times 22 \times 22 \mathrm{~mm} ; 40 \mathrm{gm})$ used for FMM experiments. (D) Time lines indicating the sequence of behaviors and cue onset-offset in the north task.

east-south-west-south-east, and so forth. Thus, there were eight possible paths between the feeders in the two versions of the task (Figure 1B). A cue illuminated either the north or south feeder, depending upon the task being performed. This cue was only visible when the monkeys had made their decision about the location of the next feeder, had turned and approached the feeder. The northsouth cue went off when the feeder lid was lifted; cues were not visible on the east and west feeders at any time. At each feeder, the monkeys had to choose to move either to the left or to the right (Figure 1B), but critical trials were movements either to the left or to the right when the monkeys were at the north or south feeders. This task depended upon memory for the previous response in the sequence in order to guide the upcoming behavioral choice, because there was no visual cue to guide the choice.

Behavioral experiments strongly suggest that task performance was mediated by memory on all trials. This conclusion was based on measurements of head movements showing that whenever a behavioral choice (a turn to the left or right) was made, head movements were typically greater than $135^{\circ}$ in order to bring the monkey's heading in the appropriate direction (Figure 2). These head turns were characterized by rapid, large amplitude movements that occurred synergistically with trunk and limb movements, resulting in a movement direction toward the next feeder where, presumably, the monkey expected to receive a reward. Head movements in the DA task were compared with those in a visually guided task in which the target feeder was selected randomly. Head movements in the random task were typically side-to-side glancing movements, larger in amplitude and significantly more frequent than those in the DA task, in which the monkey invariably made a single turn toward the correct feeder. In the FMM DA task, the monkeys typically performed at $93 \%$ correct, measured over a 60 -day period.
Of three types of errors (intrusive circling, dropping the lid, forgetting), the third, forgetting, was rare but was usually followed by recovery, which suggested knowledge about the sequential nature of the task. For example, having approached the north from the east, the monkeys would (erroneously) return to the east and open the feeder. Typically, the monkeys then circled to the west, obtained the reward (visual cues were not visible on any feeder) and continued with the sequence to the north. This demonstrates that they knew where to go after having made an error. Importantly, they avoided the north feeder, demonstrating that they remembered their previous choice. We consider the FMM DA task to be a simplified analogue of the multiple errands task (Shallice \& Burgess, 1991), in which short trips to different sites are made in order to collect expected items.

\section{Comparison of the FMM DA Task With DA Tasks in Chaired Monkeys}

In previous studies, monkeys performed the DA task while seated in a primate chair and made arm movements to three (left, center, right) response keys. To start a trial, the monkey grasped the center key and waited ( $5 \mathrm{sec})$ until the left and right keys were illuminated. A correct sequence was hold center, press left (reward), hold center, press right (reward), hold center, press left (reward), and so forth (Niki, 1974). Arm movements to the left and right were completed within $300 \mathrm{msec}$, and the return movement to the center was completed within $250 \mathrm{msec}$. An entire trial (time between rewards) averaged $5.55 \mathrm{sec}$ in the chaired experiment. In the FMM DA task, monkeys sat at each feeder (1.78 sec on average) and obtained a reward, turned and walked ( $3.7 \mathrm{sec}$ on average) to the next feeder and obtained another reward. An entire trial (time between rewards) took $5.69 \mathrm{sec}$ on average. Thus, although the FMM and chaired DA tasks differ in several respects (e.g., the freely moving monkey is 


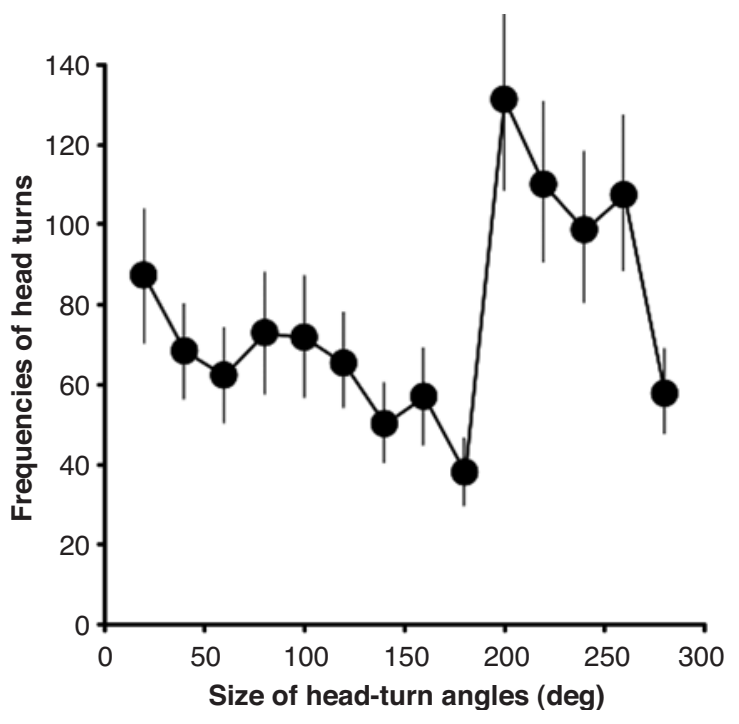

Figure 2. Measurements of angular head movements during the DA task. There is a large increase in the frequency of head movements greater than $135^{\circ}$. This is due to the nature of the DA task, because the monkeys must reorient their heads at least $135^{\circ}$ in order to move on to the next feeder. Data points (and standard deviations) are averages of head-movement measurements made over 30 days of task performance.

able to make the behavioral decision sooner; the chaired monkey is able to look at the target during the delay before the arm movement), the result of any behavioral decision is only known after a delay of approximately $5.5 \mathrm{sec}$ in both tasks.

\section{Neurophysiological Techniques}

Recordings in freely moving animals require nonstandard techniques. For example, commercial microelectrode drives (Figure 1C) are mechanically unsuitable for recordings in a situation in which the head is rapidly moving. Accordingly, we developed a 40-gm microdrive that enabled us to implant electrodes, which remained in the brain for up to 6 months (Lei et al., 2004; Wilson, Ma, Greenberg, Ryou, \& Kim, 2003). This approach influenced our sampling technique in that we concentrated on a relatively small number of electrodes that extensively sampled a limited number of trajectories over a long period of time. This allowed us to record from individual neurons in a variety of tasks and over several days (Greenberg \& Wilson, 2004), which was important, because it was not possible to record from each task every day, and thus chronically implanted electrodes allowed us to record from single cells in several tasks over several days.

\section{Techniques for Measuring Position Within the Testing Room}

The monkeys wore helmets continuously to protect electrodes and electrode drives. The helmets allowed the placement of light-emitting diode (LED) arrays used to monitor the location of the monkeys within the testing room. The LED arrays were mounted on the helmet so that the LEDs were displaced $4.5 \mathrm{in}$. left (red) and right (blue) of the center of the helmet. The locations of the LEDs were tracked by a computer interfaced with a ceiling-mounted video camera (Cheetah, Neuralynx, Inc.) at a temporal resolution of $60 \mathrm{frames} / \mathrm{sec}$ with $640 \times 480$ pixel resolution provided by the NTSC signal. The area within which the monkeys walked was represented as a $100 \times 100$ element grid where 1 pixel $=7$ sq. $\mathrm{cm}$ (PrimaTracker; Kim, Lim, Ryou, \& Wilson, 2004; available at http://w3.arizona.edu/ primate/).
The neuronal firing rate was obtained by dividing the number of spikes emitted by the amount of time spent in a particular location (pixel). The software was used to determine the center of the monkey's head (location), the direction in which its head was facing, and the speed and angular velocity of movements of its head.

\section{Data Analysis}

Files containing the timestamps of action potential occurrence and critical task events (lid opening, closing, etc.) were processed with NeuroExplorer software (Plexon, Inc.). From these data files, the neuronal firing rate on each trial and for each condition was calculated (spikes/sec).

The firing rate of each neuron was analyzed with one- and twoway analyses of variance (ANOVAs), with three time conditions (Epochs 1, 2, and 3; see Table 1) and eight paths (direction). Counts of firing rate were calculated for all three epochs. Epoch 1 began 1 sec prior to the opening of the feeder lid. In this interval, the monkey arrives at the feeder, sits and then reaches and opens the lid. Epoch 2 was the retrieval interval (averaging $1.8 \mathrm{sec}$ ) between lid opening and lid closing in which the monkey retrieves a reward. In this interval, the monkey holds the lid open, retrieves the food, and drops the lid. Epoch 3 was a 3 -sec period triggered by the closing of the feeder lid. At this point, the monkey turns and walks to the next feeder.

In order to quantify the selectivity of each neuron, two basic procedures were implemented. After identification of neurons that showed a significant effect of epoch (simplistically, a significant neuronal response before, during, or after the behavioral response of opening or closing the feeder lid), we separated the data into eight different trial types. For example, neurons were determined to be responsive in (1) the sit and reach condition, (2) the food retrieval interval, and (3) the walking condition. Within each epoch, the analysis determined whether this occurred on all eight trial types or just particular trial types. We wanted to know whether pathway/trial type (i.e., spatial direction) was an important factor for neuronal responsiveness. Thus, pairwise comparisons were used to identify significant differences among the eight directions. The results of these tests were then employed to calculate a discriminability index (DI; Mikami, Nakamura, \& Kubota, 1994) where

$$
\mathrm{DI}=\frac{\text { number }_{\text {discriminated }}}{\text { number of paths }-1}
$$

in which a large fraction indicates that the maximal response was strongly discriminative; that is, it discriminated between many of the other responses.

\section{Waveform Discrimination}

Action potentials were discriminated off line after each experiment in order to ensure that recordings were from single neurons. Software was obtained from several sources: commercial sources (Offline Sorter; Plexon, Inc.), freeware (MClust; Neuralynx, Inc.), and software written in our laboratory (B.-H. Kim).

\section{Recording Locations}

The recording chambers were implanted bilaterally, guided by prior MRI scans, X-radiographs, and a Crist MRI-compatible stereotaxic instrument aimed at a region approximately $18-32 \mathrm{~mm}$ ante-

Table 1

Interval Data for Analyses of Variance

\begin{tabular}{cccl}
\hline Epoch & Timing & Duration $(\mathrm{sec})$ & Behavior \\
\hline 1 & -1 to 0 & 1 & Sit and reach \\
2 & 0 to 1.8 & 1.8 & Retrieve \\
3 & 2 to 5 & 3 & Walking \\
\hline
\end{tabular}

Note-Zero is the time of arrival at the feeder, signaled by lid opening. 
rior to the interaural line, and between 3 and $15 \mathrm{~mm}$ lateral to the midline (areas 9 and 46). Neuronal activity, which is characteristic of oculomotor neurons in the caudal arcuate region, was not observed. Data collection is still in progress, but the recording locations have been identified by superimposing X-ray and MRI scans.

\section{RESULTS}

\section{Overt Behavior of Monkeys}

The behavior of the monkeys during DA task performance is quite stereotyped in most respects. The traces in Figure 1B show the paths taken during the recording shown in Figure 3, with 80 trials (eight paths, averaging 10 trials/path). Monkeys typically take fixed paths during task performance, taking the shortest route between the feeders; they are efficient in this respect. Figure 3 shows that the duration of time spent at the different feeders is similar $(M=1.78 \mathrm{sec})$, as are travel duration $(M=3.7 \mathrm{sec})$ and travel speed $(M=0.64 \mathrm{~m} / \mathrm{sec})$ between the feeders. Moreover, monkeys have a highly stereotyped way of opening the feeder lids: a marked preference to use the hand that is closest to the feeder on arrival. When they approach feeders on their left (paths: WN, NE, ES, SW), the left hand is used to open the lid, and the right hand is used to retrieve the food. Conversely, when they approach feeders on their right (paths: EN, NW, WS, SE), the right hand is used to open the lid, and the left hand is used to retrieve the food. Thus there is little to distinguish the monkeys' behaviors in traveling between the feeders and their behavior at the feeders. Complete details of the behavior of freely moving monkeys will be published elsewhere. In addition, the physical appearance of the feeders and the testing room is uniform in most respects; only two obvious visual cues (the door and the grill) provide information about location within the room. Accordingly, the stereotyped behaviors and homogeneous sensory environment provide a stable reference against which differences in neuronal activity can be compared; these references were helpful in determining factors that could account for the neuronal responses.

The data summarize averages (and standard deviations) from 3 different monkeys from 30 days of testing. With respect to travel times, there were slight differences between monkeys, but overall no pathway was found to be exceptionally different, except the west-to-south route. In addition, there were no substantial differences in the time spent at particular feeders. Overall, overt behavioral measures indicate that the monkeys responded to each path and feeder in much the same way; travel speeds are correspondingly similar.

\section{Selective Neuronal Activity}

From the outset, we note that dIPFC neuronal activity during the freely moving version of the DA task resembles that reported in classical studies, in that the timing of neuronal activity appears to reflect cue-delay-response activity. In addition, we emphasize that neuronal activity recorded in the freely moving version is characterized by a selectivity that is striking in view of the homogeneity of the monkeys' overt behaviors and the appearance of the testing room. It is clear that straightforward descriptions such as "move left hand," "prepare to make a right turn," or "memorize target feeder on the left" do not account for the relationship between neuronal activity and

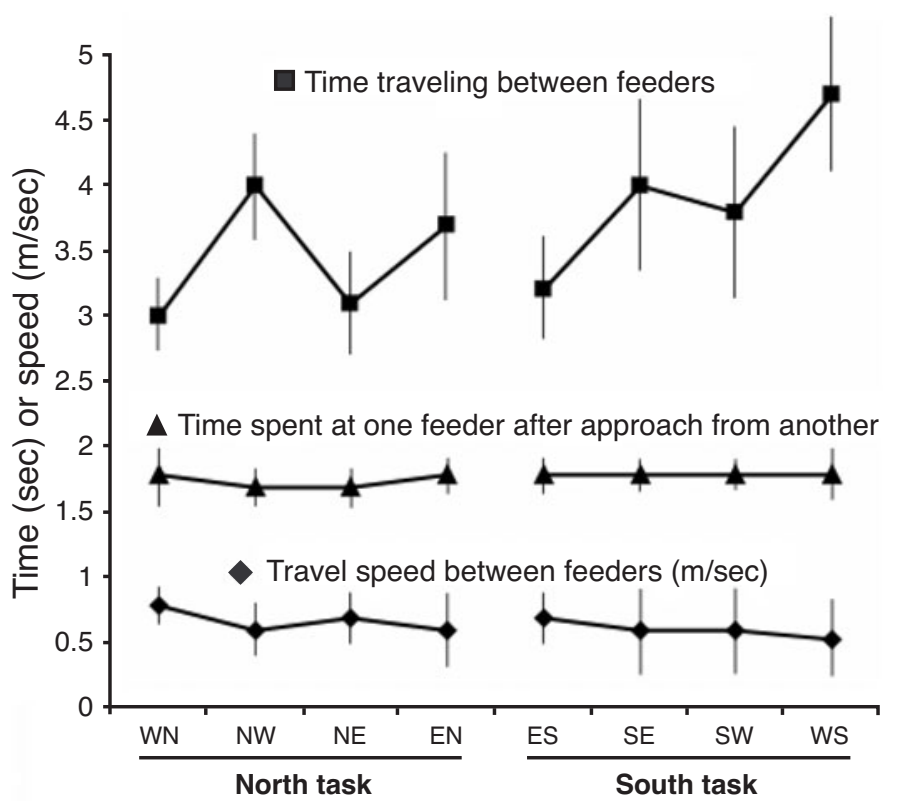

Figure 3. Times spent walking different paths and times spent at different feeders. 
behavior. At one level, the FMM version of the DA task is a two-choice task carried out at four locations. Thus simple relationships between neuronal activity and behavior should produce obvious symmetrical responses on specific types of trials. However, such simple relationships were rarely encountered. We contend that the neuronal activity reflects the cognitive organization of the task as a structured sequence rather than reflecting behavioral variables such as hand or head movements per se.

Seventy-two of 192 recorded neurons were selective (Table 2) in at least one of the three epochs. Certain neurons were selective in more than one epoch. For example, the responses of certain neurons began while the monkey was retrieving food (Epoch 2), and these responses continued as the monkey turned and moved toward the next target (Epoch 3). See Figure 7 for a different example.

Neurons that were selective for Epoch 1 (arrival) were the most common. Such neurons responded before the monkey arrived and opened the feeder. The increase in firing rate of the neuron (Figure 4A) began 2-3 sec before lid opening. The neuronal activity was selective $[F(7,207)=12.146, p<.001]$ and responded significantly more on the south-to-east path than on all other seven paths. The responses of these neurons were not related to arm or other movements (such as the postural changes involved in slowing, sitting, or reaching) in this interval. If this were so, neuronal activity related to arm movements would be identifiable by characteristic activity on all trials in which either the left (paths: WN, NE, ES, SW, or the right arm (paths: EN, NW, WS, SE) was used. The neuron shown in Figure 4A does not reflect this pattern of activity because it responds on only one of the eight possible conditions. Indeed, nonselective neurons were not common and in any event, they would not be classified as selective because they responded on all or half of the trial types. It is worth noting that the maximal and minimal responses were on the south-to-east path versus east-to-south. That is, the same path was being taken in both cases, but the neuronal responses were increases versus decreases in firing rate. Such neurons appear to be directionally selective.

A second group of selective neurons was active during Epoch 2. Figure 4B shows an example in which the recorded cell responded transiently after lid opening. These neuronal responses were selective $[F(7,234)=$ $8.648, p<.001]$; in this case, the maximal neuronal response on the east-to-south path was significantly greater

Table 2

Numbers of Selective Dorsolateral Prefrontal Neurons

\begin{tabular}{|c|c|c|c|c|c|c|c|c|c|}
\hline \multirow[b]{2}{*}{ Epoch } & \multicolumn{4}{|c|}{ North Task } & \multicolumn{4}{|c|}{ South Task } & \multirow[b]{2}{*}{ Total } \\
\hline & WNE & NWN & NEN & $\overline{\text { ENW }}$ & ESW & SES & SWS & WSE & \\
\hline 1 & 8 & 2 & 1 & 4 & 17 & 8 & 8 & 8 & 56 \\
\hline 2 & 8 & 3 & 2 & 3 & 4 & 4 & 0 & 6 & 30 \\
\hline 3 & 2 & 4 & 5 & 5 & 8 & 6 & 4 & 8 & 34 \\
\hline Total & 18 & 9 & 8 & 12 & 21 & 18 & 12 & 22 & \\
\hline
\end{tabular}

than on six of the other conditions. Such selective neurons were not related to the act of food retrieval. If so, they should be active on all trials, or differentially responsive, dependent upon the arm movements being made. The neuron shown in Figure 4B cannot be understood in terms of movements because, for example, the neuronal responses occur strongly on the ESW path and weakly on the WNE path when the left hand is used to open the feeder lids on both trial types. Moreover, the maximal and minimal responses occur on trials in which the south feeder is approached from the east and west, respectively. Thus the neuronal responses do not reflect the location of the south feeder since these responses appear to depend upon either the direction of approach or departure. As with the neuron shown in Epoch 1, it is possible that this neuronal activity is carrying directional information even though it occurs while the monkey sits at the feeder.

Neurons selective for Epoch 3 were most responsive when the monkey had begun to walk toward another feeder, indicated by closing of the feeder lid. These neurons were not related to walking. On the contrary, their activity was selective for particular paths. Figure 4C shows a neuron that is most responsive on the north-toeast path. Statistical analysis shows that the response is selective $[F(7,240)=15.73, p<.001]$. The neuronal responses on the north-to-east path are significantly greater than on all seven of the other paths. In contrast to the increase in firing rate on the north-to-east path, the response on the east-to-north path is a small decrease in firing rate below spontaneous activity. Thus, such neurons appear to carry directional information, as is suggested for the other selective neurons active in Epochs 1 and 2.

It is of interest to examine the frequency at which these selective responses occurred. We held two a priori hypotheses about such distributions. The first was that each path would be equally represented across a population of neurons; this idea is fundamental to the view that neuronal activity in the dIPFC is responsible for task performance and therefore that each component of the task must be represented in the brain in order to ensure successful performance. A second and very different hypothesis was that there would be skews in the representation of different types of neurons; this idea is predicated on the view that sensory or motor factors are the basis for neuronal selectivity. In this view, we might expect certain paths to be particularly effective in eliciting selective responses. For example, the north-east-north paths were somewhat different from the others in that allocentric cues (the bottom of the laboratory door) are potentially visible and thus could play a role in the control of performance.

The graph in Figure 5 represents, for each epoch, the number of neurons that were maximally responsive for particular paths (see also Table 2). Two major points are clear from this graph. First, more cells were selective for Epoch 1 (56) than for the other two epochs $(30,34)$, a point that is demonstrated in Table 2. The significance of this finding is unclear. Second, in most cases, examples 

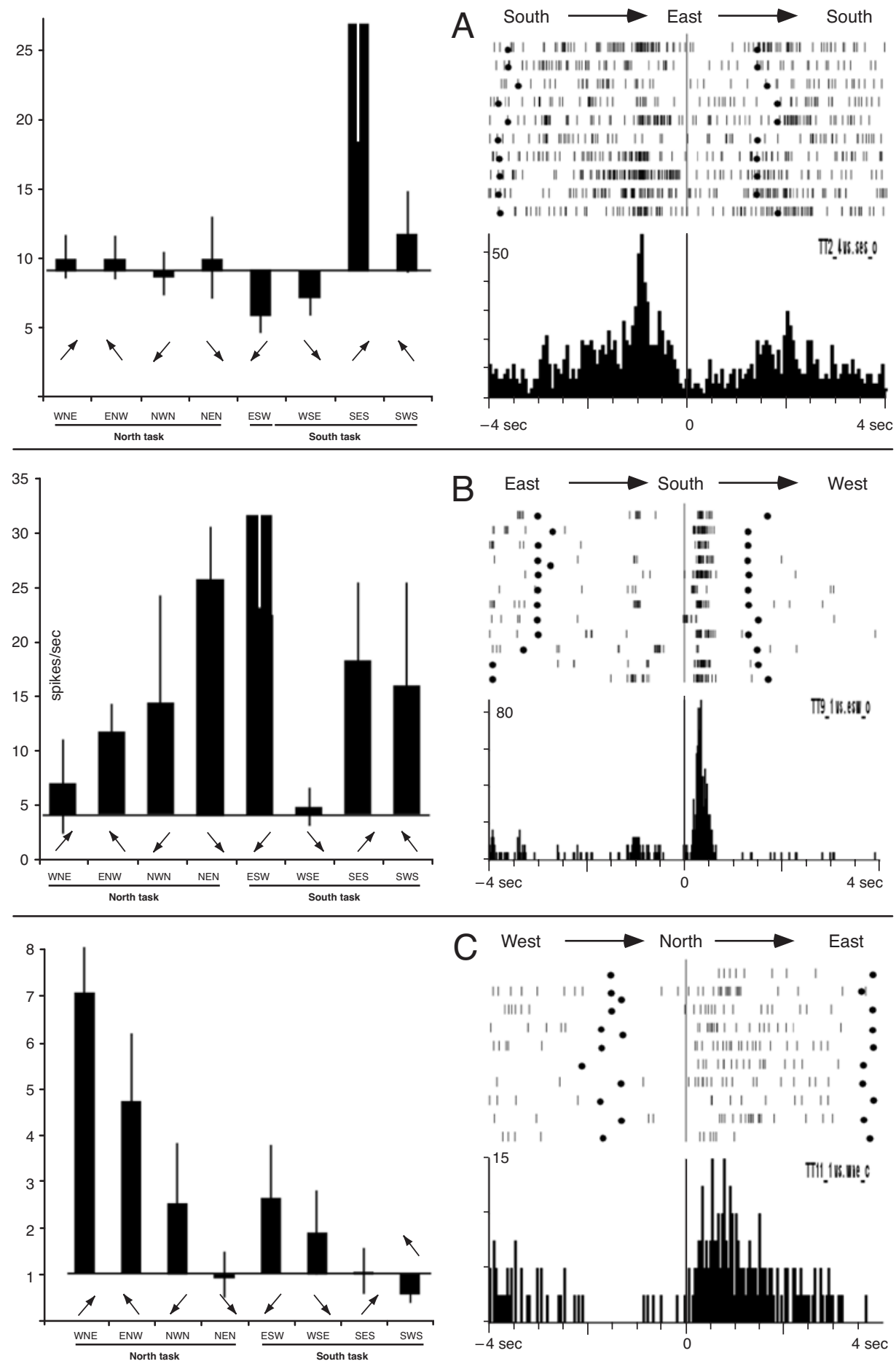

Figure 4. Three dIPFC neurons with selective activity in Epochs 1 (A), 2 (B), and 3 (C). On the left, histograms represent mean firing rates for each of the eight possible paths. The horizontal lines represent the mean firing rate of the neuron throughout the experiment; the histograms are plotted relative to mean firing rate. On the right, raster and peristimulus time histogram graphs show firing rate on the most responsive of the eight paths. Neuronal firing is aligned (time 0 ) either on the time of arrival at the feeder (A, B), or on departure from the feeder (C). $Y$-axes represent firing rate in spikes/second. Tick marks represent occurrence of action potentials; dot marks represent the opening or closing of the feeder lid. 


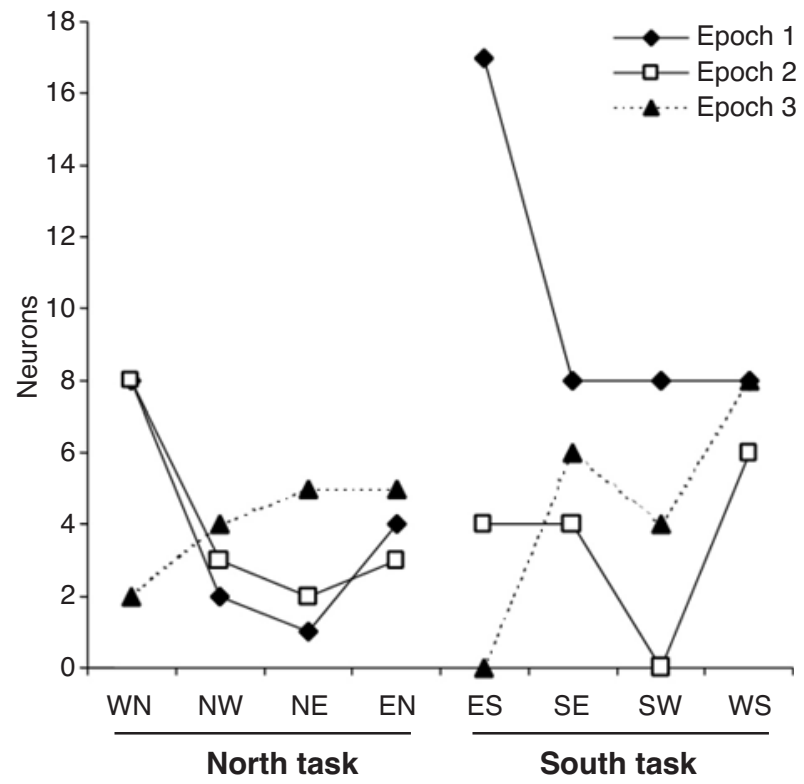

Figure 5. Frequency distribution of selective neurons in three epochs.

of selective neurons were found for each of the eight possible paths. Moreover, for each path direction there were similar proportions of neurons, and thus almost every possible type of selective response was found. The proportions of the incidence of such neurons were tested with chi-square tests. For Epoch 1, there was a significant departure from homogeneity $\left[\chi^{2}(7)=24.857, p<\right.$ $.001]$, which was due to the large number of neurons that were maximally responsive to the SWS path. This inhomogeneity did not achieve significance when the SWS data were taken from the analysis $\left[\chi^{2}(6)=10.7, p<\right.$ .097]. The data for Epoch $2\left[\chi^{2}(7)=5.9, p<.431\right]$ and Epoch $3\left[\chi^{2}(7)=4.3, p<.637\right]$ did not reach significance, indicating that the incidence of these neurons was quite homogeneous. It should be noted that there were small differences in the proportions of such cells, and while it is possible to conjecture that the differences are important, the significance of these differences will be better assessed with a larger sample, which is in progress.

However, there is one aberrant observation. An exceptionally large number of neurons were selectively responsive on the approach (Epoch 1) to the south feeder from the east. Conversely, on departure (Epoch 3) from the south box going to the west feeder, no cells were found that encoded this particular path. Also, a relatively large number of cells responded on the west-to-north path. These striking differences contrast with the largely homogeneous data obtained in overt behavior (see Figure 3 ). Whereas the behavior shows no apparent differences, the neuronal data suggest that these particular paths are different from the others. One possible explanation is that environmental cues (the floor grill) in the room are potentially visible on these paths, although it should be noted that the grill was not visible when these neurons were most responsive (i.e., the $1-\mathrm{sec}$ period before lid opening). Thus the significance of this asymmetry is not clear. This issue may be resolved as more data become available.

\section{Discriminability}

The above analysis indicates that neurons are selective for one or more of the three epochs (based on ANOVA). It was of interest to know the degree of selectivity of these neurons, and for this purpose we used an index of discriminability (Mikami et al., 1994) for each selective neuron. There were two major findings. First, the mean discriminability index was approximately the same for all three neuronal types (arrival, .45; retrieval, .51; departure, .49). These data are plotted in Figure 6. Moreover, it is apparent that the slopes of the frequency distributions for the three epochs are quite similar. Second, the average value (.48) of discriminability indicates that the maximal response is statistically discriminable from at least three of the other seven paths. Thus selective neurons tend to fire most strongly for one or two particular trial types and discriminate between three or more of the other seven conditions. This degree of discriminability occurs whether or not the neurons are selective for the arrival, retrieval, or departure from the different feeders.

\section{Neuronal Responses Occur Across Epochs}

As noted earlier, many neurons were active in two or more epochs. An example of this multiepoch neuronal activity is shown in Figure 7. The neuronal responses shown in Figure $7 \mathrm{~A}$ are selective $[F(7,63)=48.1, p<$ .0001 ; one-way ANOVA] during the performance of the FMM DA task. The maximal responses occurred on the north-to-east path (there was no significant difference with the east-to-north path), and were significantly stronger than on the other six paths. Thus this neuron was most active in the northeast quadrant of the room, irrespective of the direction of travel. In particular, there was little activity on the south-to-east and east-to-south paths. Inspection of the peristimulus histograms in Figure 7B shows that changes in neuronal activity are a function of the epoch. These data were recorded from the same neuron while the monkey performed a modification of the FMM DA task in which it alternated between the north and south feeders, guided by the presence of visual cues on these feeders. In this task, the neuron responded on the north-toeast path (Figure 7B), as it did in the FMM DA task (Figure 7A), and continued to respond as the monkey bypassed the east feeder. These responses began shortly after the lid was dropped on the north feeder and continued until the monkey's arrival at the south feeder (Epoch 3). As the monkey arrived at the south feeder, neuronal firing decreased transiently and then increased again while the monkey sat and opened the feeder (Epoch 1). Firing rate decreased before the lid was dropped, and the neuron was largely silent as the monkey walked clockwise to the north feeder. This type of differential neuronal activity resem- 


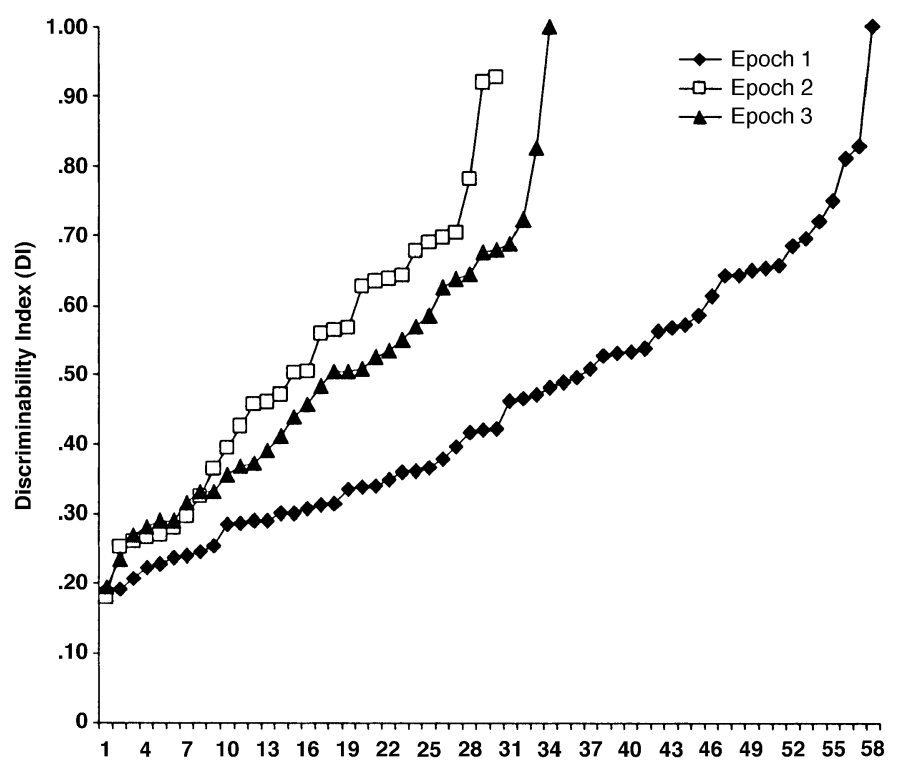

Figure 6. Incidence of the discriminability of selective dorsolateral prefrontal neurons. Each data point represents the discriminability index for a single neuron.

bles delay-related responses in conventional tasks in that the responses are sustained and differentially reflect the spatial component of the task, and the differential activity is observed both during the delay and response epochs.

\section{DISCUSSION}

In the present experiments, we found that the timing of dIPFC neuronal responses resemble those described in previous reports in monkeys. For example, Niki (1974) described spatially selective neuronal activity that incremented at the end of the delay (the equivalent of FMM Epoch 1), after the choice and before the response (FMM Epoch 2), and when the delay began (FMM Epoch 3). These similiarities are not surprising because monkeys in both paradigms are engaged in selecting a spatially directed behavioral response that must be remembered over a brief delay period in both conventional and freely moving experiments. What is surprising, however, is the degree of selectivity we observed in which neurons appear to be tuned to specific components of the task. In order to put this finding into context, we will first describe previous behavioral and neurophysiological studies and consider what one might expect to see in the freely moving DA task on the basis of these previous results. We conclude that the neurophysiological results are not solely explicable in terms of a map of spatial location but must additionally incorporate information about the sequential organization of events. Finally, we compare the recordings of single neurons with neuropsychological studies that suggest that the dIPFC represents sequences of actions.

Before proceeding, we briefly comment on the relationship between the present results and experiments in rat hippocampus that have demonstrated that single cells respond maximally as rats walk through particular locations in an extensive testing environment (e.g., O'Keefe, 1999). We compared dlPFC and hippocampal pyramidal neurons in the FMM DA task and found that place cells exist in monkey hippocampus (Kim et al., 2004; Ma et al., 2003; Wilson et al., 2003). However, such hippocampal responses are qualitatively and quantitatively different from the selective dlPFC neurons described in the present study. The distinction between the two structures appears to be that dIPFC neurons are "task-related," while the primary correlate of hippocampal pyramidal cells is that of location within the testing room. Whereas neurons in dIPFC are highly selective for epochs and direction and the neuronal responses are strongly synchronized with arrival or departure from the feeders, hippocampal pyramidal neurons do not appear to be entrained by task events such as the arrival, opening, or departure from the feeders. On the contrary, such neurons respond weakly but clearly in some parts of the testing room and are unresponsive in others (e.g., http://w3.arizona.edu/ $\sim$ primate/). In addition, we have not observed head direction cells (e.g., Taube, 1998) in the dlPFC or in the hippocampus. Note that in the DA task, each cell is tested at each location after approach from two directions. While head direction cells would be expected to respond independently of the approach direction, this is clearly not the case (e.g., Figure 4B) for the selective dIPFC neurons described in this article.

\section{Relationships With Previous Studies}

The dIPFC is intimately involved in the performance of the DA task. Although the DR task has been the more popular model for engaging spatial working memory 

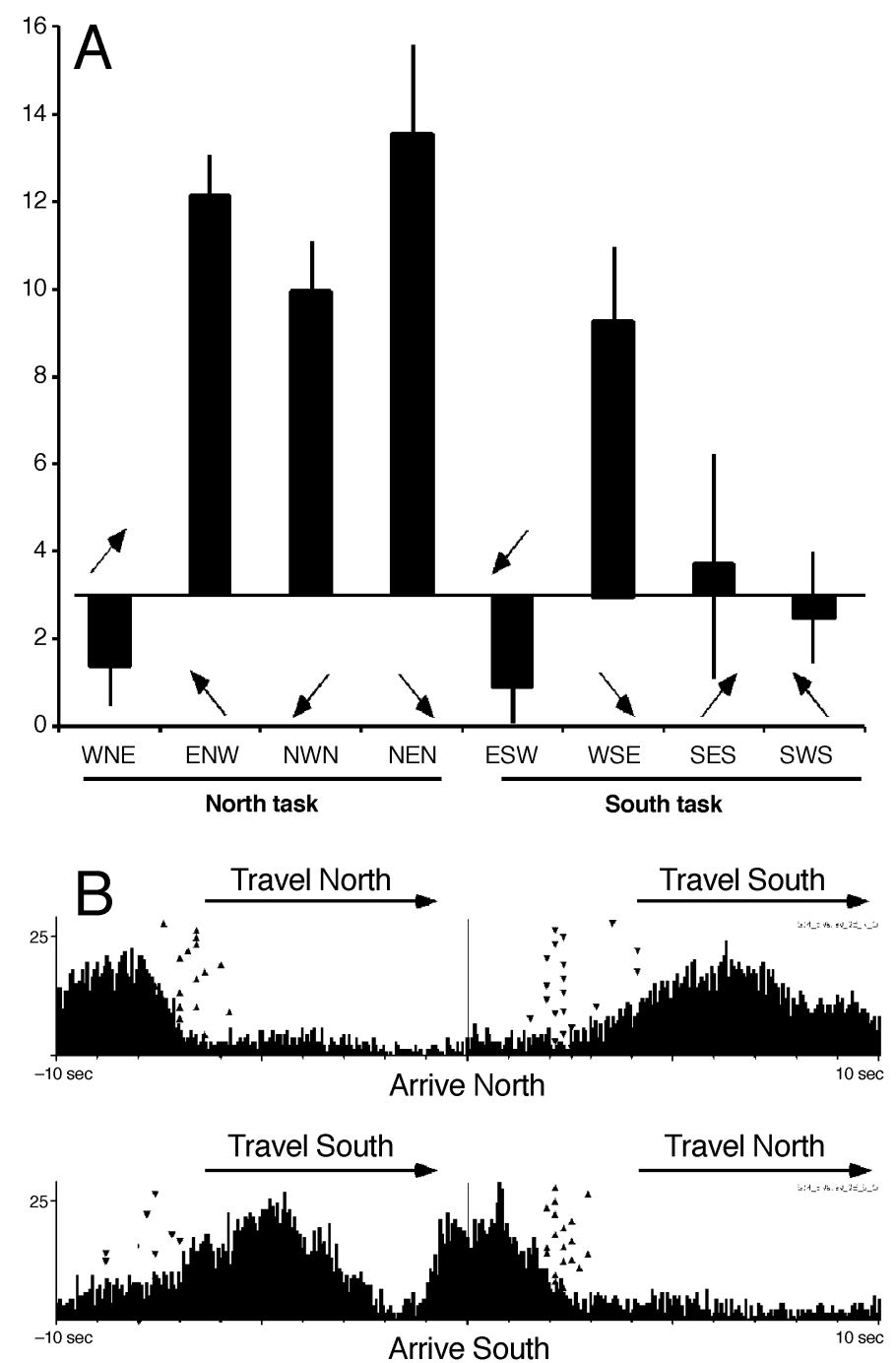

Figure 7. Differential neuronal activity in a north-south alternation task. (A) Histograms of firing rate in the eight paths of the FMM DA task. Average firing rate measured across the entire experiment is 3 spikes/sec. (B) Histograms show \pm 10 sec of neuronal activity aligned on the time of the monkey's arrival at the north and south feeders. The neuron responds differentially, increasing over a 6 -sec period as the monkey approaches the south feeder but is unresponsive as the monkey approaches the north. Triangles indicate times at which the lid was dropped. Note that the visual experience during task performance is largely identical on both head-north and head-south trials.

(Goldman-Rakic, 1987) in nonhuman primates, the DA paradigm was instrumental in the evolution of the model. In particular, four seminal studies demonstrate the importance of the principal sulcus in mediating the performance of spatial working memory tasks (Butters \& Pandya, 1969; Goldman \& Rosvold, 1970; Gross \& Weiskrantz, 1962; Mishkin, 1957) and thus were a fundamental platform on which the cognitive functions of the dIPFC were developed in the next 3 decades by Goldman-Rakic and colleagues (e.g., Levy \& Goldman-Rakic, 2000) and by Joaquin Fuster and colleagues (Fuster, 1997). In another important experiment, Stamm (1987) found that pre- frontal damage produced deficits in a locomotor version of the DA task, suggesting that the role of the dlPFC in spatial memory extends beyond the domain of arm movements. Eye movements have also been used as a measure of memory for spatial location, and imaging studies employing this technique have shown that both oculomotor DA and DR tasks engage similar networks in the prefrontal and parietal cortices and hippocampus (Friedman $\&$ Goldman-Rakic, 1994). Thus a range of studies point to the involvement of the dIPFC as an important node for mediating the performance of several forms of spatial working memory task. 
Niki and colleagues (1974) published a series of reports describing delay-related dlPFC neurons during DA performance in the 1970s (see also Carlson, Rama, Tanila, Linnankoski, \& Mansikka, 1997). Niki's DA task required the monkey to hold a response key for $5 \mathrm{sec}$, resulting in the illumination of two choice keys to the left and right of the hold key; the correct response is an alternationnamely, to press the choice key that was not selected on the previous trial. Niki described neurons that are differentially active (with respect to arm movement in the left or right direction) in the delay period some seconds before the response is made. Fuster and colleagues (Fuster, Bauer, \& Jervey, 1982) reported similar findings in the DR task.

\section{What Cognitive Processes are Necessary to Mediate the DA Task?}

In the chaired version of the DA task (Niki, 1974), monkeys were required to recall from memory the location of the previous target (or the movement necessary to acquire the target), and then to select a spatially directed response that is the alternate of the previous trial. There is considerable similarity in the general behavioral requirements between previous and present studies. Moreover, there are obvious similarities in the overall temporal form of the neuronal responses. For example, Figures 4A and $7 \mathrm{~B}$ illustrate neuronal activity that begins some seconds before the response is made (lid opening); simplistically, these observations in the FMM DA task resemble delay-related activity described in conventional versions of the DA task. These comparisons are superficial, however. We do not know if neurons recorded in the FMM DA task would also respond differentially in the chaired version of a task in which an arm or eye movement was required. Accordingly, our conclusions are necessarily limited. However, we will assume that the responses are equivalent for the time being, with the caveat that this remains to be tested.

It is possible that the performance of the FMM DA task is mediated by learning to perform a sequence of spatially directed actions in which the monkey alternates between the feeders: go to the center, go left of center, go to the center, go right of center, and so forth. Monkeys must keep track of their recent actions, their position in the sequence, the particular version (north or south) of the FMM DA task currently being performed, and the principle of alternation. While conceptually simple, this task appears to require considerable effort from the monkeys (submitted), perhaps due to the similarity between trials and, consequently, the development of proactive interference (Thompson-Schill et al., 2002).

We speculate that the following cognitive events occur during the task. Once a monkey arrives at the feeder and retrieves the reward, it must recall its previous actions and generate a new response direction on the basis of memory for the previous trial and its outcome. It is worth noting that selection of the response direction is performed very accurately and rapidly in well-trained monkeys. Measurements of their head movements show that once the feeder lid is dropped, monkeys make very large and rapid head movements that bring their bodies into line with the next target (Figure 2). Accordingly, we suppose that the decision about response direction is actually made before or at the time of dropping the lid. We infer that neuronal activity in Epochs 2 and 3 is related to the decision about selection of the response direction. If this were the case, it would be consistent with the selectivity of neuronal activity in Epochs 2 and 3 when the monkey is not walking to the next target. Once the monkey is en route, it is presumably maintaining in memory its recent actions in order to evaluate its decision when it arrives at the feeder.

\section{The High Degree of Selectivity in the FMM DA Task}

The FMM version of the DA task can be considered a two-choice (left/right), ambulatory version of the conventional DA task. Apart from the behavioral response (walking to the target, as opposed to making an arm movement), the major behavioral difference between the freely moving and conventional studies is that the FMM DA task is done at four different locations within the testing room. However, these locations and the movements between them give rise to essentially identical testing conditions in all four cases. Given the homogeneity of the testing conditions and the common alternation paradigm, one might expect that the neuronal activity in the freely moving DA task would strongly resemble that reported in chaired monkeys (Carlson et al., 1997; Niki, 1974), and superficially this expectation is confirmed by the presence of sustained activity in the "delay" interval (Figures 4 and 7). However, more searching tests show that the neuronal responses are remarkably selective. For example, consider what one might expect to see in the FMM DA experiment given the results from Niki's classic studies. Niki observed differential neuronal responses that reflected the spatial direction of the impending movement. On the basis of these findings, we might expect to find neuronal responses that occur upon departure on all four trial types when the monkey has to generate a turn to the right based on memory. However, these expectations were not confirmed. On the contrary, a striking aspect of the present data is the marked selectivity of the neurons, where each neuron appears to be tuned to a few specific components of the task as if each target feeder and each direction in which the feeder is approached were separately encoded by individual neurons. This selectivity excludes the possibility that these differential neurons encode the movements that occur on each trial, or provide a mechanism for the encoding of a general mnemonic signal such as "memorize target feeder on the left."

\section{What is Encoded by Spatially Selective Delay-Related Neuronal Activity?}

Assuming that the neurons recorded in the FMM DA tasks are of the same functional class as the delay neurons recorded in chaired monkeys (Fuster et al., 1982; Niki, 1974), this gives rise to the question of what is being en- 
coded by such delay activity. The selective nature of the present results suggests that the neurons that we recorded are not simply correlated with memory for locations to the left and right of the present location. In fact, there is evidence that this is an oversimplification even for studies using conventional methods, as described below.

Differential activity in two-choice, spatial (left, right) DA and DR tasks is usually interpreted as evidence for working memory for spatial location, a view that is supported by oculomotor variants of the DR task (Funahashi et al., 1989). However, more recent studies suggest that this interpretation requires further analysis. Other reports have shown that anticipatory delay activity encodes more than the memory for a single movement or spatial location (Barone \& Joseph, 1989; Funahashi, Inoue, \& Kubota, 1997). For example, Funahashi et al. (1997) compared the responses of dlPFC neurons in both a DR and a delayed sequential reaching (DSR) task. As expected, delay-related neurons were selectively active during the memorization of specific spatial targets, but they also found that the temporal order in which these targets were presented was important for the neuronal activity. Their example (Figure 6) shows delay-related activity in the DR task when the remembered location is on the right, thus confirming classical observations. Correspondingly, the neuron is also active when the first of the two memoranda is on the right in the DSR task. However, critical trials occur when the right location is the second item to be remembered. Figure 6 shows that the neuronal activity does not occur with this pair of sequentially presented stimuli even though memory for both items is essential for correct performance. Thus Funahashi et al.'s (1997) experiment shows that certain dIPFC neurons encode the sequential order of items to be remembered. Moreover, they show that neurons that encode sequential order may superficially appear to be a simple correlate of spatial location when recorded in a classical DR task. This important experiment thus sheds new light on spatial working memory tasks, indicating that the classical interpretation - that delay neurons simply encode spatial location-is an oversimplification brought about by the disarming simplicity of the DR task. Recent studies strongly suggest that sequential order is encoded by dlPFC neurons in nonspatial working memory tasks (Inoue \& Mikami, 2002; Ninokura, Mushiake, \& Tanji, 2003).

The data reported by Funahashi et al. (1997) are evidence that the single neurons in dIPFC encode memory for spatial location and temporal order. We suggest that this also represents a form of planning. Our results showing that dorsolateral prefrontal neurons are active at certain parts of a complex alternating sequence are fundamentally consistent with Funahashi et al. (1997) in that that the selective neuronal responses recorded in the FMM DA task cannot be accounted for simply as an encoding of the location of a target to the left or right of the subject. These studies are consistent with impairments of complex sequences of motor acts following prefrontal damage.

\section{The Notion of dIPFC as Mediating Learned Sequences}

It has been known for many years that prefrontal damage in humans impairs performance when subjects are asked to produce multiple responses in a sequence when there is no impairment in generating the individual actions that compose the sequence (see Lepage \& Richer, 1996). For example, Petrides and Milner (1982) showed that prefrontal damage resulted in a failure to organize self-initiated selection of a sequence of responses and to carry them out correctly when monitoring their execution was required. In this experiment, subjects were required to learn a novel contingency and follow it. Other research has shown that prefrontal damage can disrupt the performance of seemingly routine acts typical of everyday living (Della Malva et al., 1993; Humphreys \& Forde, 1998; Schwartz et al., 1991; Sirigu et al., 1998; Zanini, Rumiati, \& Shallice, 2002). Although Shallice and Burgess (1991) stress the importance of the prefrontal cortex in novel, nonroutine actions, other studies suggest that prefrontal damage can disrupt very simple sequences of actions such as making a cup of tea. Accordingly, it seems reasonable to infer that the performance of the FMM DA task could be implemented as a learned sequence of actions by populations of differentially selective neurons in the dIPFC.

The responses of neurons in many parts of the brain are characterized by selectivity, and this is true in the prefrontal cortex where, for example, different neurons appear to provide the components of a memory map of visual space (Funahashi et al., 1989). Recent research has given rise to the possibility that such neurons carry both spatial and temporal information, and we interpret our data in this way; the selectivity of neurons in the FMM DA task suggests that they reflect the encoding of information about sequences required for task performance.

Presumably, neuronal selectivity results from a learninglike process that functions to encode different components of a complex sequence of regularly occurring events. An important next step in the experiments will be to examine how this tuning develops. In parallel experiments, we have explored neuronal responses in monkeys that are learning relationships between stimuli and responses, and it is clear that the dIPFC is very active in go/no-go tasks in which monkeys are learning to make or withhold behavioral responses. Moreover, neurons that respond in these tasks demonstrate plasticity - that is, their responses reflect learning about new stimulus-response relations (Wilson, Greenberg, Seok, \& Ma, 2004). Thus the next step will be to explore freely moving monkeys in experiments in which learning is required. In this way, we hope to discern the mechanisms underlying neuronal selectivity.

In summary, we suggest that selective neuronal activity is tuned through learning and reflects the fundamental units of a planning mechanism where individual neurons encode specific components of a sequence of behavioral actions and their temporal order. Populations of such neurons represent all the steps necessary to perform the north and south tasks. The sustained activity of these 
neurons suggests that planning and working memory mechanisms are integrated. These results thus represent an evolutionary step based on a solid foundation of research and theory from the laboratories of GoldmanRakic and of Fuster.

\section{REFERENCES}

BAdDeley, A. D. (1996). Exploring the central executive. Quarterly Journal of Experimental Psychology, 49A, 5-28.

BARONE, P., \& JosePh, J.-P. (1989). Prefrontal cortex and spatial sequencing in macaque monkey. Experimental Brain Research, $\underline{\mathbf{7 8}}$, 447-464.

BUTTERS, N., \& PANDYA, D. (1969). Retention of delayed-alternation: Effect of selective lesions of sulcus principalis. Science, 165, 12711273.

Carlson, S., Rama, P., Tanila, H., Linnankoski, I., \& MansikKa, H. (1997). Dissociation of mnemonic coding and other functional neuronal processing in the monkey prefrontal cortex. Journal of Neurophysiology, 77, 761-774.

CaVAda, C., \& Goldman-RAKIC, P. S. (1989). Posterior parietal cortex in rhesus monkey: I. Parcellation of areas based on distinctive limbic and sensory corticocortical connections. Journal of Comparative Neurology, 287, 393-421.

Courtney, S. M., Ungerleider, L. G., Keil, K., \& Haxby, J. V. (1997). Transient and sustained activity in a distributed neural system for human working memory. Nature, 386, 608-611.

Della Malva, C. L., Stuss, D. T., D’Alton, J., \& Willmer, J. (1993). Capture errors and sequencing after frontal brain lesions. Neuropsychologia, 31, 363-372.

D'Esposito, M., \& Postle, B. R. (1999). The dependence of span and delayed-response performance on prefrontal cortex. Neuropsychologia, 37, 1303-1315.

Dombrowski, S. M., Hilgetag, C. C., \& Barbas, H. (2001). Quantitative architecture distinguishes prefrontal cortical systems in the rhesus monkey. Cerebral Cortex, 11, 975-988.

Friedman, H. R., \& Goldman-RaKic, P. S. (1994). Coactivation of prefrontal cortex and inferior parietal cortex in working memory tasks revealed by 2 DG functional mapping in the rhesus monkey. Journal of Neuroscience, 14, 2775-2788.

Funahashi, S., BRUCE, C. J., \& Goldman-Rakic, P. S. (1989). Mnemonic coding of visual space in the monkey's dorsolateral prefrontal cortex. Journal of Neurophysiology, 61, 331-349.

Funahashi, S., InOUe, M., \& Kubota, K. (1997). Delay-period activity in the primate prefrontal cortex encoding multiple spatial positions and their order of presentation. Behavioural Brain Research, 84, 203-223.

FUSTER, J. M. (1997). The prefrontal cortex: Anatomy, physiology, and neuropsychology of the frontal lobe. New York: Raven.

Fuster, J. M., BAuer, R. H., \& Jervey, J. P. (1982). Cellular discharge in the dorsolateral prefrontal cortex of the monkey in cognitive tasks. Experimental Neurology, 77, 679-694.

Goldman, P. S., \& RosvolD, H. E. (1970). Localization of function within the dorsolateral prefrontal cortex of the rhesus monkey. Experimental Neurology, 27, 291-304.

GOLDMAN-RAKIC, P. S. (1987). Circuitry of primate prefrontal cortex and regulation of behavior by representational memory. In J. M. Brookhart \&. V. B. Mountcastle (Series Eds.) \& F. Plum (Vol. Ed.), Handbook of physiology: Section 1. The nervous system: Vol. 5. Higher functions of the brain (pp. 373-417). Washington, DC: American Physiological Society.

Greenberg, P. A., \& Wilson, F. A. W. (2004). Functional stability of dorsolateral prefrontal neurons. Journal of Neurophysiology, $\mathbf{9 2}$ 1042-1055.

Gross, C. G., \& WeIsKrantz, L. (1962). Evidence for dissociation of impairment on auditory discrimination and delayed response following lateral frontal lesions in monkeys. Experimental Neurology, 5, 453-476.

HUMPHREYs, G. W., \& FordE, E. M. E. (1998). Disordered action schema and action disorganization syndrome. Cognitive Neuropsychology, 15, 771-811.

INOUE, M., \& Mikami, A. (2002). Delay period activity of the primate prefrontal cortex during the serial probe reproduction task. Society for Neuroscience Abstracts, 28, 676.13.

JACOBSEN, C. F. (1935). Functions of the frontal association areas in primates. Archives of Neurology \& Psychiatry, 33, 558-569.

KIm, B.-H., Lim, S.-L., Ryou, J.-W., \& Wilson, F. A. W. (2004). Taskrelated remapping of hippocampal place fields in freely moving monkeys. Society for Neuroscience Abstracts, 30, 1007.2

Lei, Y., Sun, N., Wilson, F. A. W., Wang, X., Chen, N., Yang, J., Peng, Y., Wang, J., Tian, S., Wang, M., MiaO, Y., XhU, W., Qi, H., \& MA, Y. Y. (2004). Telemetric recordings of single neuron activity and visual scenes in monkeys walking in an open field. Journal of Neuroscience Methods, 135, 35-41.

LEPAGE, M., \& RiCHER, F. (1996). Inter-response interference contributes to the sequencing deficit in frontal lobe lesions. Brain, 119, 1289-1295.

LeVY, R., \& Goldman-Rakic, P. S. (2000). Segregation of working memory functions within the dorsolateral prefrontal cortex. Experimental Brain Research, 133, 23-32.

MA, Y.-Y., RYOU, J.-W., KIM, B.-H., \& Wilson, F. A. W. (2003). Spatially directed movement and neuronal activity in freely moving monkeys. In S. Mori, D. G. Stuart, \& M. Wiesendanger (Eds.), Brain mechanisms for the integration of posture and movement (Progress in Brain Research, Vol. 143, pp. 505-512). Amsterdam: Elsevier.

MaLmo, R. B. (1942). Interference factors in delayed response in monkeys after removal of frontal lobes. Journal of Neurophysiology, 5, 295-308.

Mikami, A., Nakamura, K., \& Kubota, K. (1994). Neuronal responses to photographs in the superior temporal sulcus of the rhesus monkey. Behavioural Brain Research, 60, 1-13.

MisHKIN, M. (1957). Effects of small frontal lesion on delayed alternation in monkeys. Journal of Neurophysiology, 20, 615-622.

NiKI, H. (1974). Prefrontal unit activity during delayed alternation in the monkey: 1. Relation to direction of response. Brain Research, $\mathbf{6 8}$, 185-196.

NinOKURA, Y., MushiaKe, H., \& TANJI, J. (2003). Representation of the temporal order of visual objects in the primate lateral prefrontal cortex. Journal of Neurophysiology, 89, 2868-2873.

O'KeEFE, J. (1999). Do hippocampal pyramidal cells signal non-spatial as well as spatial information? Hippocampus, 9, 352-364.

Ó Scalaidhe, S. P., Wilson, F. A. W., \& Goldman-RAKIC, P. S. (1999). Face selective neurons during passive viewing and working memory performance of rhesus monkeys: Evidence for intrinsic specialization of neuronal coding. Cerebral Cortex, 9, 459-475.

Petrides, M., \& Milner, B. (1982). Deficits on subject-ordered tasks after frontal- and temporal-lobe lesions in man. Neuropsychologia, 20, 249-262.

RAJKOWSKA, G., \& Goldman-RaKIC, P. S. (1995). Cytoarchitectonic definition of prefrontal areas in the normal human cortex: I. Remapping of areas 9 and 46 using quantitative criteria. Cerebral Cortex, $\mathbf{5}$, 307-322.

Schwartz, M. F., Reed, E. S., Montgomery, M. W., Palmer, C., \& MAYER, M. H. (1991). The quantitative description of action disorganization after brain damage: A case study. Cognitive Neuropsychology, 8, 381-414.

SeRENO, A. B., \& Holzman, P. S. (1995). Antisaccades and smooth pursuit eye movements in schizophrenia. Biological Psychiatry, 37, 394-401.

ShAllice, T., \& Burgess, P. W. (1991). Deficits in strategy application following frontal lobe damage in man. Brain, 114, 727-741.

Sirigu, A., Cohen, L., Zalla, T., Pradat-Diehl, P., Van EeckHout, P., Grafman, J., \& AGID, Y. (1998). Distinct frontal regions for processing sentence syntax and story grammar. Cortex, 34, 771-778.

Smith, E. E., \& Jonides, J. (1999). Storage and executive processes in the frontal lobes. Science, 283, 1657-1661.

STAMm, J. S. (1987). The riddle of the monkey's delayed-response deficit has been solved. In E. Perecman (Ed.), The frontal lobes revisited (pp. 73-89). New York: IRBN Press. 
Stuss, D. T., \& Benson, D. F. (1986). The frontal lobes. New York: Raven. TAube, J. S. (1998). Head direction cells and the neurophysiological basis for a sense of direction. Progress in Neurobiology, 55, 225-256.

Thompson-Schill, S. L., Jonides, J., Marschuetz, C., SMITH, E. E., D’Esposito, M., Kan, I. P., KNight, R. T., \& Swick, D. (2002). Effects of frontal lobe damage on interference effects in working memory. Cognitive, Affective, \& Behavioral Neuroscience, 2, 109-120.

Wilson, F. A. W., Ma, Y.-Y., GreenberG, P. A., Ryou, J.-W., \& Kim, B.-H. (2003). A microelectrode drive for long-term recording of neurons in freely moving and chaired monkeys. Journal of Neuroscience Methods, 27, 49-61.

Wilson, F. A. W., Greenberg, P. A., Seok, B., \& MA, Y.-Y. (2004).
Learning-related responses in go/no-go neurons in dorsolateral prefrontal cortex. Society for Neuroscience Abstracts, 32, 324.5.

WiLson, F. A. W., KIM, B.-H., RYoU, J.-W., \& MA, Y.-Y. (in press). An automated food-delivery system for behavioral and neurophysiological studies of learning and memory in freely moving monkeys. Behavior Research Methods, Instruments, \& Computers.

Zanini, S., Rumiati, R. I., \& Shallice, T. (2002). Action sequencing deficit following frontal lobe lesion. Neurocase, 8, 88-99.

(Manuscript received August 23, 2004; revision accepted for publication December 17, 2004.) 